\title{
Ueber ein Papillom der Conjunctiva mit ausgedehnter Bildung von Becherzellen.
}

\author{
Von \\ Professor Dr. A. Wagenmann \\ in Jena.
}

Hierzu Tafel XII, Fig. 1-2.

Erst in neuerer Zeit hat man den an der Conjunctiva vorkommenden polypenartigen Neubildungen nähere Aufmerksamkeit geschenkt und sie nach pathologisch-anatomischen Gesichtspunkten zu classificiren gesucht. Wie Elschnig ${ }^{1}$ ) hervorhebt, sind echte Polypen der Bindehaut, d. h. "gestielt vorragende hyperplastische Wucherungen einer streng umschriebenen Stelle der Schleimhaut durch ihre ganze Dicke hindurch, also sämmtlicher Schleimhautbestandtheile" bisher noch gar nicht durch genauere Untersuchung nachgewiesen worden. Das, was uns klinisch als polypenartige Neubildungen der Schleimhaut entgegentritt, kann einen ganz verschiedenen histologischen Charakter haben. Wir wissen jetzt, dass, abgesehen von den z. B. nach Traumen aus Granulationsgewebe entstandenen Wucherungen, weiche und harte tuberöse Fibrome, Papillome, Adenome und auch Sarcome im ersten Stadium in Polypenform sich entwickeln können ${ }^{2}$ ).

1) Ueber die polypenähnlichen Geschwülste der Bindehaut. Arch. für Augenheilkunde XIX, 1889, p. 63.

2) Vergleiche folgende Arbeiten, in denen auch die nähere 
Ich möchte nun im Folgenden die kurze Beschreibung eines derartigen als gestielter Polyp klinisch in Erscheinung getretenen Conjunctivaltumors mittheilen, der pathologischanatomisch den gestielten Papillomen zuzuzählen ist und der wegen einiger histologischer Besonderheiten einiges Interesse erregen dürfte.

Der Trmor stammt ron einem 50jährigen Manne, der sich am 29. März 1892 in der Heidelberger Universitäts-Augenklinik vorstellte, da er erst seit wenigen Monaten die rasch wachsende Geschwulst bemerkt hatte. Klinisch stellte sich die Neubildung: dar als ein mit einem dünnen Stiel implantirter Schleimhautpolyp, der von der Mitte der rechten unteren Uebergangsfalte ansgegangen war, und der die Grösse und Forn einer kleimen Bohne besass.

Bei der gewölnlichen Blickrichtung der Augen war der Tumor von dem unteren Lid bedeckt und machte sich nur dureh eine geringe Prominenz desselben bemerkbar. Beim Blick nach oben and bei schnelleren Augenbewegungen trat er spontan zu Tage und ectropionirte das untere Lid. Er besass eine stark röthliche Färbung und schien reelt gefässreich zn sein. An seiner glatten Oberfläche erkannte man besonder's schön bei Loupenhetrachtung äberall in regelnässigen $\Lambda$ bständen kleine rothe Pünktchen, ron denen jedes ron einem grauröthlichen Hof ungelıen war, sorlass die Obertläche dadurch in regelmässige Felder getheilt erschien. Der kurzo Schleimhautstiel enthielt eine Anzalıl grösserer Gefässchen, die sich ron unten her in die Geselwulst einsenkten. Die Conjunctira des Auges war etwas injieirt und serernirto ziemlich reichliel.

In Vertretung meines damaligen Chefs, Herrn Professor Leber, entfernte ich die Geschwulst, indem ich den Stiel mit einer Arterienpincette abklemmte, mit einem Doppelfaden unterhalb der Pincette durchstach, nach beiden Seiten unterband und

Literatur zu finden ist. Parisotti: Contribution à l'étude des tuneurs bénignes de la conjunctive. Recueil d'Ophth. 1884, p. 575 . Elschnig: Ueber die polypenähnlichen Geschwülste der Bindehaut. Arehiv für Augenheilk. XIX, 1889, p. 63. S. Fuchs: Ueber das Papillom der Conjunctiva. Archiv für Augenheilkunde Bd. XX, 1889, p. 416. - Schirmer: Ueber Adenome der Karunkelgegend nebst einem neuen Fall. v. Graefe's Archiv f. Ophth. XXXVII. 1. p. 216. Rumschewitsch: Zur Onkologie der Conjunctiva. Klin. Monatsblättor für Augenheilkunde 1891, p. $260 \mathrm{ff}$. 
dann oberhalb der Ligatur durchschnitt. Die kleine Wunde heilte glatt, nachılem die Fäden nach zwei Tagen entfernt worden waren. Der Mann stellte sich innerhalb der nächsten Monate melnfach vor, ohne dass ein Recidiv beobachtet wurde.

Der mir von Herm Professor Leber zur Untersuchung: freundlichst überlassene 'Tumor' wurde in Müller'scher Flüssignkeit gehärtet, in Alkohol nachgehärtet, in Celloidin eingebettet und parallel zum Verlauf und Eintritt des Stiels geschnitten. Bei der Nachhärtung in Alkohol veränderte sich die bis dahin glatte Oberfläche der Geschwulst derart, dass die Peripherie der kleinen oben erwähnten Felder sich etwas zurückzog, wodurch der Tumor ganz das Aussehen einer Himbeere erhielt.

Bei der mikroskopischen Untersuchung erkennt man, dass der Tumor aus den Verästelungen der durch den Stiel in ihn eintretenden Gefässe aufgebant ist, indem je ein oder melrere bis etwa vier dicht zusammenliegende Gefässstämmchen von einem dicken Mantel von Epithelzellen umgeben sind. Die sieh verzweigenden Getässe geben gewissermassen nur das Gerüst der Geschwulst $a b$, an das sich die die Hauptmasse der Geschwulst bildenden Zellmäntel anlehnen. Verfolgt man den Stiel des Tumors nach innen zu, so erkennt man, dass seine Gefässe sich schnell in kleine Stämmchen auflösen, dass das dieselben einhüllende Bindegewebe rasch abnimmt und die im Tumor befindlichen Gefässe nur in äusserst spärlicher Menge begleitet, und dass die kleinen Aestchen sich schnell mit einem dicken Zellmantel ungeben haben. Die in der Geschwulst sich verzweigenden Gefässe haben vorzugsweise, aber keineswegs ausschliesslich, eine radiäre Riehtung; vielfach kann man constatiren, dass sie in der Geschwulst nach den verschiedensten Richtungen hin seitliche Aeste abgeben. Dadurch trifft man in jedem Schnitte quer-, längs- und schräggetroffene Gefässe an.

Das den Stiel überkleidende Conjunctivalepithel nimmt nach der Geschwulst an Mächtigkeit rasch zu und geht direct in das Epithel der periphersten Stränge über. Die an der Oberfläche liegenden Zellen werden etwas grösser und besitzen ebenso wie die Deckzellen der Geschwulst die Gestalt von kurzen, breiten Cylinderzellen.

Da die erwähnte Bezichung der Epithelzellen zu den Gefässen besteht, scheint die Geschwulst auf dem Durchschnitt aus lauter Läppchen zu bestehen (T. XII, Fig. 1). Ihr Aussehen erinnert lebhaft an das gewisser Angiosarcome. Beachtenswerth ist ferner, dass die Läppchen vielfach ein für sich abgeschlossenes 
Ganzes bilden und nicht confluiren, sondern nur sich innig berühren und sich isoliren lassen. Ich komme darauf später noch zurück.

Die einzelnen Theile der Geselnwulst bieten nun noch weitere zum Theil auffallende Besonderheiten dar.

Zunächst zeichnete sich die innen gelegene Parthie der Stränge, also die Gefässschicht, durch mannigfache Verschiedenheiten und Complicationen mit secundären Veründerungen aus. In einer Reihe ron Läppchen findet sich in der Mitte nur ein einziges Gefäss, oft mit relativ beträchtlichem Lumen, dessen Wand aus einem einfachen Endotheirohr besteht, das den Epithelzellen unmittelbar aufliegt. Andere Aestchen besitzen zwischen Endothel und Zellenmantel eine zarte, aus fibrillärem Bindegewebe bestehende Wand. In vielen Läppchen sind die Gefässe von etwas reichlicheren Bindegewebsziıgen umgeben; gewölnlich liegen dann auch melirere Stämmchen in der Mitte eines Strangs, olne dass man aber etwa Unterscheidungen in Arterien und Venen machen könnte. Von glatten Muskelfasern ist an keinem einzigen Gefäss der Geschwulst etwas zu erkennen (T. XII, Fig. 2).

Das die Gefässe ungebende Bindegewebe ist äusserst arm an Zellen, fein fibrillär, vielfach ron hoinogenem, hyalinem Aussehen. $A b$ und zu kommen einzelne Lymphzellen darin vor. Die äusserst zarten Fibrillen sind rielfach zu den Gefässen concentrisch rerlaufend, so dass man nicht weiss, wie weit sie zur Gefässwand selbst gehören. An anderen Stellen zweifelt man, ob man nicht nur Degenerationsvorgänge der Gefässwand dureh Wucherung der Endothelien mit fibrillärer und lyaliner Entartung ror sich hat, zumal dort, wo thatsächlieh derartige Endothelwucherungen rorkommen. Vielfach wird man ganz an die Veränderungen erinnert, die in Angiosarcomen vorkornmen und mehrfach beschrieben sind.

Wie eben erwälnt, finden sich an einer Reihe ron Gefässen deutliche Proliferationsrorgänge der Wand und alle möglichen Stadien und Uebergänge bis zur vollständigen Obliteration der Lumina. Zuweilen erkennt man in den Verdickungen noch Reste von Endothelien. Ist das Gefäss rollkommen obliterirt, so ist das Centrum der Läppchen in eine feintibrilläre oder homogenhyaline Masse umgewandelt, an der man hier und da weiter einen feinkörnigen Zerfall wahrnehmen kann. Offenbar hängen diese Veränderungen mit dem Alter der Gefässe zusammen, weshalh man sie auch nur in der Mitte der Geschwulst, in den ältesten Partlien antrifft. 
Der die Gefässe umkleidende, meist reeht dicke Zellenmantel besteht aus Epithelzellen, die eine radiäre Anordnung erkennen lassen. Die innerste an die Gefässschicht stossende Zellenlage wird durehweg von schmalen, nicht sehr hohen Cylinderzellen mit länglichen ovoiden Kernen gebildet. Da die Zellen eine geringe Breite besitzen, liegen die Kerne dicht zusammen. Die daran stossenden Schichten bestehen aus grössexen, polymorphen, meist kugeligen Zellen mit rundlichen Kernen und grösserem Protoplasmaleib, so dass die Kerne hier weiter anseinander gerîekt sind (T. XII, Fig. 2). Ihre Färbbarkeit nimmt nach aussen zu ab; wälrend die unmittelbar an die Gefässe stossenden Kerne sich intensiv und gleichmässigg färben, werden sie nach der Peripherie zu inmer blasser und besitzen nur ein sich intensiver färbendes Netzwerk im Innern. Dort, wo die Stränge ein in sich abgeschlossenes Ganzes bilden und gewissermaassen begrenzte Epithelzapfen darstellen, die sich mit den benachbarten nur innig berühren, nimmt die äusserste Zellenschicht das Aussehen von Deckzellen an, die einen regelmässigen Ueberzug des Mantels bilden. Diese Zellen besitzen die Gestalt ron kurzen Cylinderzellen, in denen die Kerne stets auf der inneren Seite der Zelle liegen. Dieselben Zellen finden sich anch überall dort, wo die Stränge die freie Oberfläche der Geschwulst erreichen. In grosser Ausdelmung sind nun diese Deckzellen in Becherzellen umgewandelt. Die einzelne Zelle ist, wie bei der gewölnlichen Umwandlung in Becherzellen stets zu sehen ist, bedeutend rergrössert, vor allem in der Höhenausdehnung; der Kern nimmt, meist vollkommen plattgedrückt, die tiefste Stelle der Zelle ein; der Inhalt hat ein hyalin-glasiges Aussehen. Doch erkennt man meist ein feines Netzwerk in dem Zellenleib (T. XII, Fig. 2).

Färbt man mit Carmin oder mit ganz schwacher Hämatoxylinlösung, so behălt der Inhalt das hyaline Aussehen und bleibt ungefärbt, während der an die Wand gedrückte Kern gefärbt ist. Färbt man in gewöhnlicher Hämatoxylinlösung, so wird der Inhalt ebenfalls stark blau gefärlbt und lässt sich nur schwer von dem meist etwas intensiver gefärbten Kern abgrenzen. Eosin färbt den Zelleninhalt nicht. Dagegen bekomnnt man sehr schöne Roth- oder Violettiärbung der Zelle hei Färbung mit Thionin, während die Kerne sich blau färben. Auch mit Fuchsin nimmt der Inhalt intensive Rothfirlumg an.

Da die oberste Lage der Deckzellen auf grosse Strecken durchweg in Becherzellen umgewandelt ist, hat die Geschwulst 
ein höchst eigenthümliches Aussehen bekommen. Die einzelnen Epithelstränge sind häufig vollkommen von einer auf dem Durchsehnitt bandförmigen Lage reihenförmig gestellter Becherzellen überzogen (Taf. XII, Fig. 1). Und dort, wo sich die Geschwulstläppchen isolirt laben und mit den benachbarten nur berühren, stossen die äusseren Oberflächen zweier benachbarter Becherzellenbeläge direct zusammen. Besonders in den mit Eosin-Hämatoxylin in der gewöhnlichen Weise gefärbten Präparaten ist das Aussehen der Geschwulst höchst merkwürdig, da the blau gefurbten Bänder dieselbe in einzelne Felder theilen (T. XII, Fig. 1). An einzelnen Theilen des Tumors ist der Saum der Becherzellen unterbrochen, da nur einzelne Zellen umgewandelt sind. Man trifft aber die Züge von Becherzellen überall in Aer Geschwulst an, auch in den tiefsten Parthien.

Wie schon erwähnt, bilden die einzelnen Zellenstränge vielfach für sich abgeschlossene Gebilde, die sich mit den benachbarten nur innig berihren, besonders in dem peripheren Theile des Tumors. Dadurch bestehen zwischen ihnen feine Gänge, die mehrfach durch die Härtung etwas breiter geworden sind als sie wohl im Leben waren. Die Gänge münden an der Obertläehe frei nach aussen. Ab und zu erkennt man darin etwas Blut, das wahrscheinlich erst bei der Operation eingedrungen ist. Ueberall dort, wo der Becherzellenbelag zu finden ist, kommen schmale structurlose Fäden vor, die sich mit Hämatoxylin blan färhen und als Schleimfäden anzusehen sind. In flen tieferen Schichten sieht man breitere Schleimschichten auftreten und vollends in den innersten Parthien kommen breite Züge von Selleimfaiden, die stellenweise anch mit massenhaften rothen Blutkörverchen und einzelnen Lymphzellen untermischt sind, vor. Auch trifft man hier ab und zu kleine Hohlräume an, die mit hyalinen Tropfen und Detritus erfüllt sind.

Dic Schleimbillung muss bei diesem massenhaiten Vorkommen von Becherzellen eine reichliche gewesen sein. Die Schleimmassen werden vermuthlich zum grossen Theil durch die vielen zwischen den Läppchen gelegenen und an der Oberfläche mündenđen Günge nach aussen befördert sein.

In dem Epithel des Bindehautstiels, das, wie erwähnt, nach dem 'Tumor zu verdickt ist, kommen ebenfalls zahlreiche Becherzellen in den oberfächlichsten Schichten vor. Das Gewebe des Stiels zeigt an einzelnen Stellen entzündliche Veränderungen in Gestalt streifenförmiger dichter Ansammlungen von Leukoeythen. 
Wie ich schon Eingangs angeführt habe, möchte ich diese polypenartige Bindehautgeschwulst auf Grund der anatomischen Untersuchung als gestieltes Papillom bezeichnen. Klinisch hätte man sie freilich schwerlich als solches erkannt, da sie im Gegensatz zu den bisher beschriebenen Papillomen eine glatte Oberfläche besass und einen bohnenförmigen gestielten Tumor darstellte. Bei der anatomischen Untersuchung jedoch constatiren wir, dass die Geschwulst die Merkmale des Papilloms besitzt: axiale Gefässe in einer wenn auch nur spärlichen Bindegewebsneubildung, umgeben von einem Mantel von Epithelzellen. Die das Gerüst bildenden Gefässe verästeln sich vielfach innerhalb der Geschwulst, so dass man wohl von einem dendritischen Papillom sprechen könnte. Immerhin aber bilden die Verzweigungen sammt den Zellenmänteln abgeschlossene Zapfen oder Läppchen, was hauptsächlich durch die Umwandlung der Deckzellen in Becherzellen zum Ausdruck kommt. Es ist eben, wie der Befund zeigt, innerhalb dex Geschwulst eine ganz beträchtliche Oberflächenentwickelung vorhanden. Im Leben war zwar der lappige Bau dadurch verdeckt, dass die die äussere Oberfläche erreichenden Läppchen innig zusammenlagen, nach aussen abgeflacht waren und dasselbe Niveau besassen. Erst bei der Alcoholhärtung wurde durch geringe Schrumpfung der die Gefässe umgebenden Theile die Oberfläche uneben, so dass der Tumor einer Himbeere glich.

Das Papillom, oder wie es Virchow nannte, das Fibroma papillare, kommt an der Conjunctiva in mehreren Formen vor, die anatomisch sämmtlich den charakteristischen Bau haben: axiales Gefäss mit Bindegewebswucherung, umgeben von einem Epithelmantel, dessen Dicke verschieden sein kann.

Saemisch ${ }^{1}$ ) unterschied das Fibroma papillare von

1) Graefe-Sämisch IV, I, p. 152. 
der Warzenbildung. Elschnig ${ }^{1}$ ) fasst beide unter dem Namen Papillom zusammen, indem er angiebt, dass die Papillome einen bald mehr zottigen, bald mehr warzigen Habitus darböten; im ersten Fall hätten wir es mit mehr himbeerartigen Geschwülstchen zu thun, im letzteren Fall mit Geschwiilstchen, die den spitzen Condylomen gleich zu halten wären. S. Fuchs, der auch betont, dass die $\mathrm{Pa}$ pillome sämmtlich zu den Fibromen gehören, prïcisirt die Formen noch näher, indem er sie mit dem Standort und der Genese in Zusammenhang bringt. Er hebt herror, dass, wenn die Papillombildung sich an einen Theil der Conjunctiva, der schon normal Papillen hat, anschliesst, die Erkrankung gewohnlich multipel auftritt und zu beetartigen Geschwülsten mit breiter Basis führt, während die Papillome der Conjunctiva bulbi und des Fornix vielästige, oft gestielte Vegetationen von papillärer Oberfläche und oft mit polypoider Form darstellen. Als dritte Form würde sich dann die diffuse Papillombildung der Conjunctiva anschliessen, von der er einen hochgradigen Fall bei diffuser Papillomatose der Cutis mittheilt.

Der von mir mitgetheilte Tumor würde zu den solitären, polypoiden, gestielten Papillomen der Uebergangsfalte gehören. Besonders bemerkenswerth ist das iberaus massenhafte Auftreten von Becherzellen in allen Theilen der Geschwulst, dessen Erklärung nicht sicher zu geben ist, wenn wir auch durch die neuesten Untersuchungen Green's ') über die Bedeutung der Becherzellen der Conjunctiva als bewiesen erachten dürfen, dass sie normale, Schleim producirende Gebilde der Bindehaut sind. Möglicherweise hat der catarrhalische Zustand, in dem sich die ganze Schleimhaut befand, auf die massenhafte Bildung einen ursächlichen Einfluss gehabt, vielleicht auch der Umstand, dass

1) 1. c. p. 74 .

2) v. Graefe's Arch. f. Opth. XL. 1., S. 1 ff.

v. Graefe's Archiv für Ophthalnologie. XL. \&. 
258 A. Wagenmann. Ueber ein Papillom der Conjunctiva etc.

die Geschwulst das untere Lid leicht spontan ectropionirte und dann frei zu Tage trat, wodurch sie mancherlei Reizen ausgesetzt war.

Erklärung der Abbildungen auf Tafel XII, Fig. 1-2.

Fig. 1. Uebersichtspräparat (schwache Vergrösserung). Die im Innern der Läppchen liegenden Gefässe sind von einem dicken Epithelmantel umgeben, dessen äusserste Lage auf grosse Strecken in Becherzellen umgewandelt ist. Die Becherzellen bilden auf dem Durchsehnitt Bänder, die die Geschwilst durchziehen (Hämatoxylin-Eosinfürbung).

$$
\begin{aligned}
& G=\text { Gefässe. } \\
& E=\text { Epithelzellen. } \\
& B=\text { Becherzellen. }
\end{aligned}
$$

Fig. 2. Ein Läppchen stark vergrössert. Die Gefüsse sind hier in ein vollkommen hyalines Gewebe eingeschlossen. Die peripherste Zellenschicht fast rings herum in Becherzellen umgewandelt. (Hämatoxylin - Eosinfărbung).

$H=$ Hyalines Gewebe.

$G=$ Gefässe.

$C=$ Querverlaufende Capillare.

$L=$ Leukocyten, in einer Spalte liegend.

$E_{I}=$ Epithelzellen in der nalchsten Umgebung der Gefässe, cylinderförmig mit stark gefärbtem Kern.

$E_{I I}=$ Epithelzellen, die Hauptmasse des Mantels bildend.

$B=$ Becherzellen, deren Inhalt mit Hämatoxylin stark blau gefärbt ist.

$K=$ Kerne der Becherzellen, die tiefste Stelle der Zelle einnehmend, vielfach platt gedrückt. 

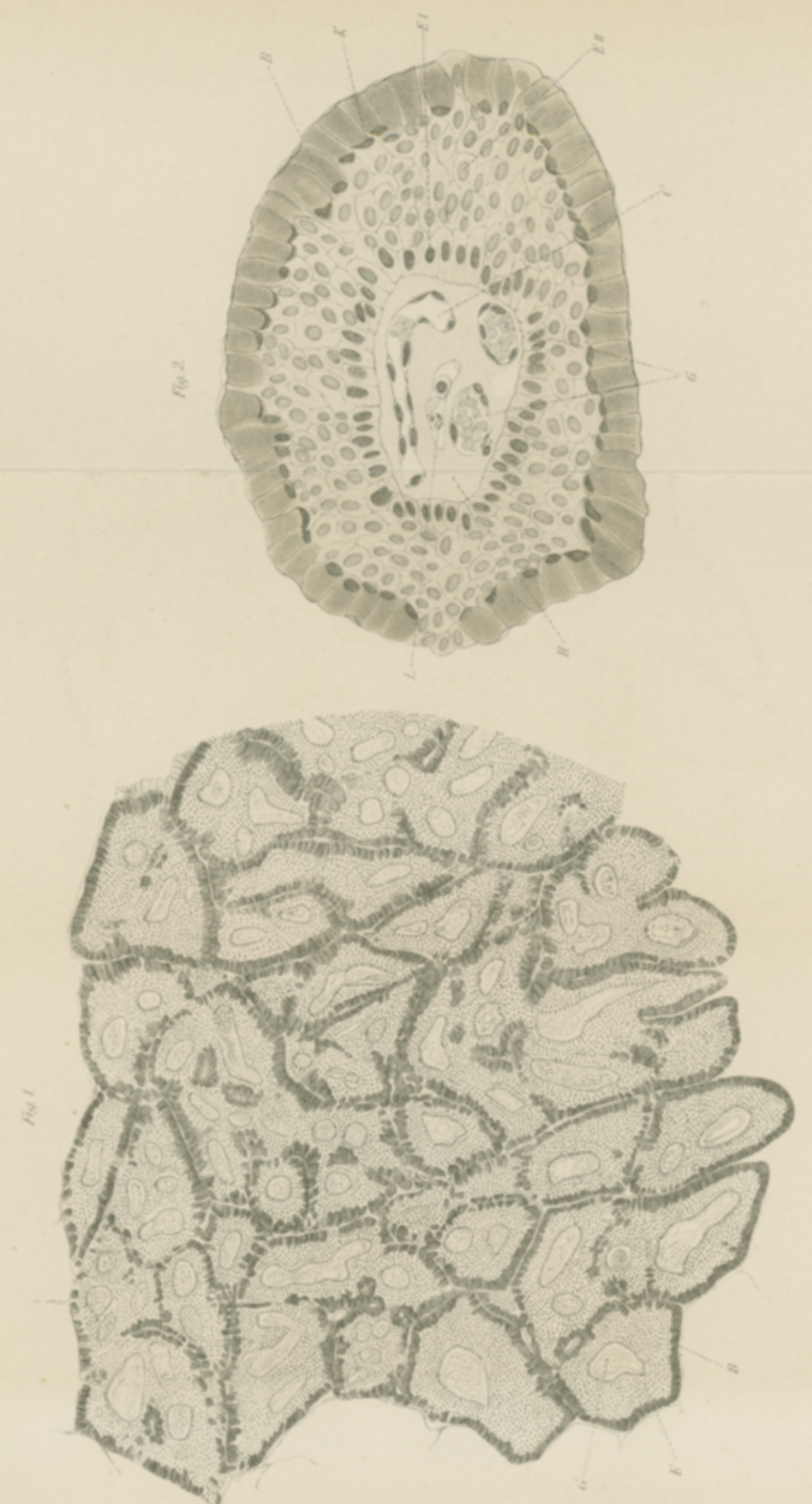